\title{
PROBABILISTIC DYNAMICS OF TWO-LAYER GEOPHYSICAL FLOWS
}

\author{
IGOR CHUESHOV, JINQIAO DUAN, AND BJÖRN SCHMALFUSS
}

\begin{abstract}
The two-layer quasigeostrophic flow model is an intermidiate system between the single-layer 2D barotropic flow model and the continuously stratified, 3D baroclinic flow model. This model is widely used to investigate basic mechanisms in geophysical flows, such as baroclinic effects, the Gulf Stream and subtropical gyres. The wind forcing acts only on the top layer. We consider the two-layer quasigeostrophic model under stochastic wind forcing. We first transformed this system into a coupled system of random partial differential equations and then show that the asymptotic probabilistic dynamics of this system depends only on the top fluid layer. Namely, in the probability sense and asymptotically, the dynamics of the two-layer quasigeostrophic fluid system is determinied by the top fluid layer, or, the bottom fluid layer is slaved by the top fluid layer. This conclusion is true provided that the Wiener process and the fluid parameters satisfy a certain condition. In particular, this latter condition is satisfied when the trace of the covariance operator of the Wiener process is controled by a certain upper bound, and the Ekman constant $r$ is sufficiently large. Note that the generalized time derivative of the Wiener process models the fluctuating part of the wind stress forcing on the top fluid layer, and the Ekman constant $r$ measures the rate for vorticity decay due to the friction in the bottom Ekman layer.
\end{abstract}

Date: March 31, 2001.

2000 Mathematics Subject Classification. Primary 60H15, 76U05; Secondary 86A05, 34D35.

Key words and phrases. Stochastic geophysical flow models, convergence in probability, random dynamical systems, random wind forcing. 


\section{INTRODUCTION}

The continuously stratified, three dimensional (3D) baroclinic quasigeostrophic flow model describes large scale geophysical fluid motions in the atmosphere and oceans. This model is much simpler than the primitive flow model or rotating Navier-Stokes flow model. When the fluid density is approximately constant, this model reduces to the barotropic, single-layer, two dimensional (2D) quasigeostrophic model. The two-layer quasigeostrophic flow model, in which the fluid consists of two homogeneous fluid layers of uniform but distinct densities $\rho_{1}$ and $\rho_{2}$, is an intermidiate system between the single-layer $2 \mathrm{D}$ barotropic flow model and the continuously stratified, 3D baroclinic flow model.

The two-layer quasigeostrophic flow model has been used as a theoretical and numerical model to understand basic mechanisms, such as baroclinic effects [29], wind-drven circulation [4, 5], the Gulf Stream [24], fluid stability [3] and subtropical gyres [30, 28], in large scale geophysical flows.

We consider the two-layer quasigeostrophic flow model ([29], p. 423):

$$
\begin{aligned}
& q_{1 t}+J\left(\psi_{1}, q_{1}+\beta y\right)=\nu \Delta^{2} \psi_{1}+f+\dot{W}, \\
& q_{2 t}+J\left(\psi_{2}, q_{2}+\beta y\right)=\nu \Delta^{2} \psi_{2}-r \Delta \psi_{2},
\end{aligned}
$$

where potential vorticities $q_{1}(x, y, t), q_{2}(x, y, t)$ for the top layer and the bottom layer are defined via stream functions $\psi_{1}(x, y, t), \psi_{2}(x, y, t)$, respectively,

$$
\begin{aligned}
& q_{1}=\Delta \psi_{1}-F_{1} \cdot\left(\psi_{1}-\psi_{2}\right), \\
& q_{2}=\Delta \psi_{2}-F_{2} \cdot\left(\psi_{2}-\psi_{1}\right) .
\end{aligned}
$$

Here $x, y$ are Cartesian coordinates in zonal (east), meridional (north) directions, respectively; $(x, y) \in O:=(0, L) \times(0, L)$, where $L$ is a positive number; $F_{1}, F_{2}$ are positive constants defined by (see also [31], p.87)

$$
\begin{gathered}
F_{1}=\frac{f_{0}^{2}}{g h_{1}} \frac{\rho_{0}}{\rho_{2}-\rho_{1}}, \\
F_{2}=\frac{f_{0}^{2}}{g h_{2}} \frac{\rho_{0}}{\rho_{2}-\rho_{1}},
\end{gathered}
$$

with $g$ the gravitational acceleration; $h_{1}, h_{2}$ the depth of top and bottom layers, $\rho_{1}, \rho_{2}$ the densities $\left(\rho_{2}>\rho_{1}\right)$ of top and bottom layers, respectively; and $L, \rho_{0}$ the characteristic scales for horizontal length and density of the flows, respectively; $f_{0}+\beta y$ (with $f_{0}, \beta$ constants) is the Coriolis parameter and $\beta$ is the meridional gradient of the Coriolis parameter, and $\nu>0$ is viscosity.. Note that $r=f_{0} \frac{\delta_{E}}{2\left(h_{1}+h_{2}\right)}$ is the Ekman constant ( [30], p.29) which measures the intensity of friction at the bottom boundary layer (the socalled Ekman layer) or the rate for vorticity decay due to the friction in the Ekman layer. Here $\delta_{E}=\sqrt{2 \nu / f_{0}}$ is the Ekman layer thickness (29], p.188). Moreover, $J(h, g)=h_{x} g_{y}-h_{y} g_{x}$ is the Jacobi operator and $\Delta=\partial_{x x}+\partial_{y y}$ is 
the Laplacian operator. Finally, $f(x, y, t)$ is the mean (deterministic) wind forcing with average zero: $\int_{O} f d O=0$

An important part of the above equation is the white noise term $\dot{W}$, describing the fluctuating part of the external forcing in the top fluid layer, see Hasselmann [22] and Arnold [2]. An example of these short time scale influences is the weather variability or wind forcing. A white noise is given as the generalized time derivative of a Wiener process $W(t)$ in a function space.

We assume periodic boundary conditions for $\psi$ in $x$ and $y$ with period $L$. In addition, we impose that

$$
\int_{O} \psi d O=0
$$

We also assume an appropriate initial condition

$$
q(x, y, 0)=q_{0}(x, y) .
$$

Stochastically forced QGE has been used to investigate various phenomena in geophysical flows [23, 27, 33, 21, 15, 9]. Recently Salmon [32] introduced some generalized two-layer ocean flow models.

It is our aim to study the long time dynamics of the stochastic differential equation with random coefficients but without white noise which we obtain after a random coordinate transformation. The solution of this differential equation generates a random dynamical system. The structure of this differential equation enables us to prove the dissipativity of the system. In order to investigate the long time dynamics we will apply the method of determining functionals in a version for random dynamical systems which is based on the convergence in probability, see Chueshov et. al [13]. It follows that the asymptotic long time behavior is determined by the asymptotic behavior of finitely many functionals. For instance, for these functionals we can choose the Fourier modes given by the eigenfunctions of the Laplacian. According to this property we can further show that the functionals defined on the top fluid layer alone can determine the asymptotical behavior of the complete two-layer system, when the fluid parameters and the Wiener process satisfy a certain condition, such as the Ekman constant $r$ is sufficiently large and the trace of the covariance operator of the Wiener process is controled by some upper bound. Note that the generalized time derivative of the Wiener process models the fluctuating part of the wind stress forcing on the top fluid layer, and the Ekman constant $r$ measures the rate for vorticity decay due to the friction in the bottom Ekman layer.

We first recall some basic facts in random dynamical systems in Section 2. In Section 3, we establish the well-posedness of the stochastic two-layer quasigeostrophic model by transforming it into a coupled system of random partial differential equations. The main results on asymptotic probabilistic determining functionals are presented in Sections 4 and 5. Finally we summarize our conclusions in Section 6. 


\section{RANDOM DYNAMICAL SYSTEM AND DETERMINING FUNCTIONALS}

It is our goal to study the long time dynamics of (11) which is influenced by random forces. Appropriate tools to treat this equation are given by the theory of random dynamical systems.

A random dynamical system consists of two components. The first component is a metric dynamical system $(\Omega, \mathcal{F}, \mathbb{P}, \theta)$ as a model for a noise, where $(\Omega, \mathcal{F}, \mathbb{P})$ is a probability space and $\theta$ is a $\mathcal{F} \otimes \mathcal{B}(\mathbb{R}), \mathcal{F}$ measurable flow: we have

$$
\theta_{0}=\mathrm{id}, \quad \theta_{t+\tau}=\theta_{t} \circ \theta_{\tau}=: \theta_{t} \theta_{\tau}
$$

for $t, \tau \in \mathbb{R}$. The measure $\mathbb{P}$ is supposed to be ergodic with respect to $\theta$. The second component of a random dynamical system is a $\mathcal{B}\left(\mathbb{R}^{+}\right) \otimes \mathcal{F} \otimes$ $\mathcal{B}(H), \mathcal{B}(H)$-measurable mapping $\varphi$ satisfying the cocycle property

$$
\varphi(t+\tau, \omega, x)=\varphi\left(t, \theta_{\tau} \omega, \varphi(\tau, \omega, x)\right), \quad \varphi(0, \omega, x)=x,
$$

where the phase space $H$ is a separable metric space and $x$ is chosen arbitrarily in $H$. We will denote this random dynamical system by symbol $\varphi$. A standard model for such a noise $\theta$ is the two-sided Brownian motion: Let $U$ be a separable Hilbert space. We consider the probability space

$$
\left(C_{0}(\mathbb{R}, U), \mathcal{B}\left(C_{0}(\mathbb{R}, U)\right), \mathbb{P}\right)
$$

where $C_{0}(\mathbb{R}, U)$ is the Fréchet space of continuous functions on $\mathbb{R}$ of uniform convergence on compact intervals which are zero at zero and $\mathcal{B}\left(C_{0}(\mathbb{R}, U)\right)$ is the corresponding Borel $\sigma$-algebra. Suppose that we have a covariance operator $Q$ on $U$. Then $\mathbb{P}$ denotes the Wiener measure with respect to $Q$. Note that $\mathbb{P}$ is ergodic with respect to the flow

$$
\theta_{t} \omega=\omega(\cdot+t)-\omega(t), \quad \text { for } \omega \in C_{0}(\mathbb{R}, U)
$$

which is called the Wiener shift.

A main source of a random dynamical system is a random differential equation. For example, let us consider the following evolution equation in some Hilbert space

$$
\frac{d u}{d t}=F\left(u, \theta_{t} \omega\right), \quad u(0)=x,
$$

over some metric dynamical system $(\Omega, \mathcal{F}, \mathbb{P}, \theta)$. If (四) is well-posed for every $\omega \in \Omega$ and solutions $u(t, \omega ; x)$ depends measurably on $(t, \omega, x)$, then the operator

$$
\varphi:(t, \omega, x) \rightarrow u(t, \omega ; x)
$$

defines a random dynamical system (cocycle) $\varphi$. For detailed presentation of random dynamical systems we refer to the monograph by L. Arnold [1].

Motivated by deterministic dynamical systems we introduce several useful notions from the theory of random dynamical systems.

An $\omega$ depending closed set $B$ contained in the separable Hilbert space $H$ is called random if the mapping $\omega \rightarrow \sup _{x \in B(\omega)}\|x-y\|_{H}$ is a random variable 
for any $y \in H$.

A random dynamical system is called a dissipative if there exists a compact random set $B$ which is forward invariant:

$$
\varphi(t, \omega, B(\omega)) \subset B\left(\theta_{t} \omega\right), t>0,
$$

and which is absorbing: for any random variable $x(\omega) \in H$ there exists a $t_{x}(\omega)>0$ such that if $t \geq t_{x}(\omega)$

$$
\varphi(t, \omega, x(\omega)) \in B\left(\theta_{t} \omega\right) .
$$

For the following we need tempered random variable: A random variable $x$ is called tempered if

$$
t \rightarrow\left|x\left(\theta_{t} \omega\right)\right|
$$

is only subexponentially growing:

$$
\limsup _{t \rightarrow \pm \infty} \frac{\log ^{+}\left|x\left(\theta_{t} \omega\right)\right|}{|t|}=0 \quad \text { a.s. }
$$

This condition is not a very strong restriction because the only alternative is that the above limsup is $\infty$ which describes the degenerated case of stationarity, see Arnold [1], page $164 \mathrm{f}$.

Our main purpose will be to estimate the degree of freedom of the long time dynamics of a random dynamical systems which stem from the two-layer flow problem of the ocean introduced above. We will apply the theory of determining functionals to estimate the degree of freedom. Since dynamics of this model is influenced by random forces we will apply the theory of determining functionals related to the convergence in probability.

We now give our basic definition:

Definition 2.1. Let $V$ be a Banach space which is continuously embedded into $H$. Assume that there exists $\tau>0$ such that $\varphi(t, \omega, x) \in L_{l o c}^{2}(\tau,+\infty ; V)$ for almost all $\omega \in \Omega$ and $x \in H$. A set $\mathcal{L}=\left\{l_{j}, j=1, \cdots, N\right\}$ of linear continuous and linearly independent functionals on $V$ is called asymptotically determining in probability if

$$
(\mathbb{P}) \lim _{t \rightarrow \infty} \int_{t}^{t+1} \max _{j}\left|l_{j}\left(\varphi\left(\tau, \omega, x_{1}(\omega)\right)-\varphi\left(\tau, \omega, x_{2}(\omega)\right)\right)\right|^{2} d \tau=0
$$

for two initial conditions $x_{1}(\omega), x_{2}(\omega) \in H$ implies

$$
(\mathbb{P}) \lim _{t \rightarrow \infty}\left\|\varphi\left(t, \omega, x_{1}(\omega)\right)-\varphi\left(t, \omega, x_{2}(\omega)\right)\right\|_{H}=0 .
$$

The theory of determining functionals was started with the papers 19 and [26] devoted to $2 D$ Navier-Stokes equations. Now this theory is welldeveloped for the deterministic systems (see, e.g., [7, 10, 11, 18, 20, 25] and the references therein). Some results are also available for stochastic systems (see, e.g., [8, 12, 13, 17]). One of the main advantages for this theory is the possibility to localize spatial domains or parameters which are 
responsible for long-time dynamics. The existence of a finite number of determining functionals means that the long-time behaviour of the system is finite-dimensional. Moreover the values of these functionals on solutions can be intertreted as a result of some measurament of the system. From applied point of view the finiteness of number of determining functionals means that we need only a finite number of devices to observe completely all dynamics of the system.

In this paper we rely on the following result.

Theorem 2.2. Assume that a random dynamical system $\varphi$ has an absorbing forward invariant random set $B$ in $V$ such that $\sup _{x \in B(\omega)}\|x\|_{V}^{2}$ is bounded by a tempered random variable and $t \rightarrow \sup _{x \in B\left(\theta_{t} \omega\right)}\|x\|_{V}^{2}$ is locally integrable. Let $\mathcal{L}=\left\{l_{j}: j=1, \ldots, N\right\}$ be a set of linear continuous and linearly independent functionals on $V$. Suppose there exist constants $c_{\mathcal{L}}>0$ and $C_{\mathcal{L}}>0$ and a measurable function $l_{\mathcal{L}}\left(x_{1}, x_{2}, \omega\right)$ which maps $V \times V \times \Omega$ in $\mathbb{R}$ such that $l_{\mathcal{L}}\left(x_{1}, x_{2}, \omega\right) \geq-c_{\mathcal{L}}$ uniformly with respect to $\left(x_{1}, x_{2}, \omega\right) \in V \times V \times \Omega$ and for $x_{1}(\omega), x_{2}(\omega) \in B(\omega)$ we have

$$
\begin{aligned}
V(t, \omega)-V(s, \omega) & \leq C_{\mathcal{L}} \cdot \int_{s}^{t} \mathcal{N}_{\mathcal{L}}(\tau, \omega) d \tau \\
& +\int_{s}^{t} l_{\mathcal{L}}\left(\varphi\left(\tau, \omega, x_{1}\right), \varphi\left(\tau, \omega, x_{2}\right), \theta_{\tau} \omega\right) \cdot V(\tau, \omega) d \tau
\end{aligned}
$$

for all $t \geq s \geq 0$, where

$$
\begin{aligned}
V(t, \omega) & =\left\|\varphi\left(t, \omega, x_{1}\right)-\varphi\left(t, \omega, x_{2}\right)\right\|_{H}^{2}, \\
\mathcal{N}_{\mathcal{L}}(t, \omega) & =\max _{j=1, \ldots, N}\left|l_{j}\left(\varphi\left(t, \omega, x_{1}\right)-\varphi\left(t, \omega, x_{2}\right)\right)\right|^{2} .
\end{aligned}
$$

Assume that

$$
\frac{1}{t} \mathbb{E}\left\{\sup _{x_{1}, x_{2} \in B(\omega)} \int_{0}^{t} l_{\mathcal{L}}\left(\varphi\left(\tau, \omega, x_{1}\right), \varphi\left(\tau, \omega, x_{2}\right), \theta_{\tau} \omega\right) d \tau\right\}<0
$$

for some $t>0$. Then $\mathcal{L}$ is a set of asymptotically determining functionals in probability for random dynamical system $\varphi$.

Proof. It is easy to find from relation (5) that

$$
V(t, \omega) \leq V(0, \omega) e^{\int_{0}^{t} l_{\mathcal{L}}(s, \omega) d s}+C_{\mathcal{L}} \int_{0}^{t} \mathcal{N}_{\mathcal{L}}(\tau, \omega) e^{\int_{\tau}^{t} l_{\mathcal{L}}(s, \omega) d s} d \tau
$$

where $l(t, \omega)=l_{\mathcal{L}}\left(\varphi\left(t, \omega, x_{1}\right), \varphi\left(t, \omega, x_{2}\right), \theta_{t} \omega\right)$. Therefore we can apply the argument given in the proof of Theorem 2.2 [13.

In the next section, we return to the two-layer quasigeostrophic flow model.

\section{Mathematical Setup AND Well-POSEDNESS OF THE RANDOM TWO-LAYER FLOWS}

In the following, $L_{\text {per }}^{2}, H_{\text {per }}^{s}$ for $s \in \mathbb{R}$ are the standard Sobolev spaces of $L$-periodic functions with the zero mean value, i.e. $\int_{O} \psi d O=0$. Let $(\cdot, \cdot)_{0}$ 
and $\|\cdot\|_{0}$ denote the standard scalar product and norm in $L_{p e r}^{2}$, respectively. Every element $u(x, y) \in L_{p e r}^{2}$ can be represented in the form

$$
u(x, y)=\sum_{j \in \mathbb{Z}^{2}, j \neq 0} u_{j} L^{-1} \exp \left\{i \frac{2 \pi}{L}\left(j_{1} x+j_{2} y\right)\right\},
$$

where the Fourier coefficients $u_{j}$ possess the property $\bar{u}_{j}=u_{-j}$ (bar denotes the complex conjugation) and

$$
\|u\|_{0}^{2}:=\int_{O}|u|^{2} d O \equiv \sum_{j \in \mathbb{Z}^{2}, j \neq 0}\left|u_{j}\right|^{2}<\infty .
$$

Note that $L^{-1} \exp \left\{i \frac{2 \pi}{L}\left(j_{1} x+j_{2} y\right)\right\}$ is the eigenelement of the $-\Delta$ with the eigenvalue $\lambda_{1} \cdot\left(j_{1}^{2}+j_{2}^{2}\right)$, where $\lambda_{1}=\left(\frac{2 \pi}{L}\right)^{2}$ is the smallest eigenvalue. The norm in $H_{\text {per }}^{s}$ is defined by the formula

$$
\|u\|_{s}^{2}:=\int_{O}\left|(-\Delta)^{\frac{s}{2}} u\right|^{2} d O \equiv \lambda_{1}^{s} \sum_{j \in \mathbb{Z}^{2}, j \neq 0}\left(j_{1}^{2}+j_{2}^{2}\right)^{s}\left|u_{j}\right|^{2}
$$

It is clear that

$$
\|\nabla u\|_{s}^{2}:=\left\|\partial_{x} u\right\|_{s}^{2}+\left\|\partial_{y} u\right\|_{s}^{2}=\|u\|_{1+s}^{2}, \quad s \in \mathbb{R} .
$$

We also denote $\mathbf{L}_{\text {per }}^{2}=L_{\text {per }}^{2} \times L_{\text {per }}^{2}$ and $\mathbf{H}_{\text {per }}^{s}=H_{\text {per }}^{s} \times H_{\text {per }}^{s}$.

We work on the phase space $\mathbf{H}_{p e r}^{-1}$ with the scalar product

$$
(q, \bar{q})_{*}=h_{1}\left(\nabla \psi_{1}, \nabla \bar{\psi}_{1}\right)_{0}+h_{2}\left(\nabla \psi_{2}, \nabla \bar{\psi}_{2}\right)_{0}+p\left(\psi_{1}-\psi_{2}, \bar{\psi}_{1}-\bar{\psi}_{2}\right)_{0},
$$

where $q=\left(q_{1}, q_{2}\right), \bar{q}=\left(\bar{q}_{1}, \bar{q}_{2}\right)$ and $\psi=\left(\psi_{1}, \psi_{2}\right) \in \mathbf{H}_{\text {per }}^{1}, \bar{\psi}=\left(\bar{\psi}_{1}, \bar{\psi}_{2}\right)$. The relation between $q$ (resp. $\bar{q}$ ) and $\psi$ (resp. $\bar{\psi}$ ) is defined by (2). Here we also use the notation

$$
p=\frac{f_{0}^{2}}{g} \frac{\rho_{0}}{\rho_{2}-\rho_{1}} .
$$

Note that $F_{1} h_{1}=F_{2} h_{2}=p$. The norm induced by this scalar product

$$
\|q\|_{*}^{2}=(q, q)_{*}=h_{1}\left\|\nabla \psi_{1}\right\|_{0}^{2}+h_{2}\left\|\nabla \psi_{2}\right\|_{0}^{2}+p\left\|\psi_{1}-\psi_{2}\right\|_{0}^{2}
$$

is equivalent to the usual norm on $\mathbf{H}_{p e r}^{-1}$. Moreover, we have the estimate

$$
h_{1}\left\|\nabla \psi_{1}\right\|_{0}^{2}+h_{2}\left\|\nabla \psi_{2}\right\|_{0}^{2} \leq\|q\|_{*}^{2} \leq a_{0}\left(h_{1}\left\|\nabla \psi_{1}\right\|_{0}^{2}+h_{2}\left\|\nabla \psi_{2}\right\|_{0}^{2}\right)
$$

where

$$
a_{0}=1+\frac{2 p}{\lambda_{1} \min \left\{h_{1}, h_{2}\right\}}=1+\frac{2}{\lambda_{1}} \max \left\{F_{1}, F_{2}\right\} .
$$

To treat the nonlinearity we need the following lemma:

Lemma 3.1. The operator Jacobian verifies, for $u, v, w$ in $H_{p e r}^{1}$

$$
\begin{aligned}
& J(u, v)=-J(v, u), \quad(J(u, v), v)_{0}=0, \\
& (J(u, v), w)_{0}=(J(v, w), u)_{0} .
\end{aligned}
$$


Moreover the following estimates hold:

$$
\begin{gathered}
\left|(J(u, v), \Delta u)_{0}\right| \leq c_{0}\|\Delta v\|_{0} \cdot\|\nabla u\|_{0} \cdot\|\Delta u\|_{0}, \quad u, v \in H_{p e r}^{2} \\
\left|(J(u, v), w)_{0}\right| \leq c_{1}\|\Delta u\|_{0} \cdot\|\Delta v\|_{0} \cdot\|w\|_{0}, \quad u, v \in H_{p e r}^{2}, w \in L_{p e r}^{2} \\
\left|(J(u, v), w)_{0}\right| \leq c_{1}\|\nabla u\|_{0} \cdot\|\Delta v\|_{0} \cdot\|\nabla w\|_{0}, \quad u, w \in H_{p e r}^{1}, v \in H_{p e r}^{2} .
\end{gathered}
$$

Here above $c_{0}=2+(\sqrt{2} \cdot \pi)^{-1}$ and $c_{1}=c_{0} \lambda_{1}^{-\frac{1}{2}}$.

Proof. We start with (10). It is easy to see that

$$
\begin{aligned}
J(u, v) \cdot \Delta u & =\frac{1}{2} \cdot v_{y}\left\{\partial_{x}\left(u_{x}^{2}-u_{y}^{2}\right)+2 \partial_{y}\left(u_{x} u_{y}\right)\right\} \\
& +\frac{1}{2} \cdot v_{x}\left\{\partial_{y}\left(u_{x}^{2}-u_{y}^{2}\right)-2 \partial_{x}\left(u_{x} u_{y}\right)\right\} .
\end{aligned}
$$

Consequently relying on property (6) we have

$$
\begin{aligned}
\left|(J(u, v), \Delta u)_{0}\right| & \leq \frac{1}{2} \cdot\left\|v_{y}\right\|_{1}\left(\left\|\partial_{x}\left(u_{x}^{2}-u_{y}^{2}\right)\right\|_{-1}+2\left\|\partial_{y}\left(u_{x} u_{y}\right)\right\|_{-1}\right) \\
& +\frac{1}{2} \cdot\left\|v_{x}\right\|_{1}\left(\left\|\partial_{y}\left(u_{x}^{2}-u_{y}^{2}\right)\right\|_{-1}+2\left\|\partial_{x}\left(u_{x} u_{y}\right)\right\|_{-1}\right) \\
& \leq \frac{1}{2} \cdot\left(\left\|v_{x}\right\|_{1}+\left\|v_{y}\right\|_{1}\right) \cdot\left(\left\|u_{x}\right\|_{L^{4}}^{2}+\left\|u_{y}\right\|_{L^{4}}^{2}+2\left\|u_{x} u_{y}\right\|_{0}\right) \\
& \leq\left(\left\|v_{x}\right\|_{1}+\left\|v_{y}\right\|_{1}\right) \cdot\left(\left\|u_{x}\right\|_{L^{4}}^{2}+\left\|u_{y}\right\|_{L^{4}}^{2}\right) \\
& \leq \sqrt{2} \cdot\|\Delta v\|_{0} \cdot\left(\left\|u_{x}\right\|_{L^{4}}^{2}+\left\|u_{y}\right\|_{L^{4}}^{2}\right) .
\end{aligned}
$$

Using the inequality (see, e.g., [18])

$$
\|u\|_{L^{4}} \leq a_{1} \cdot\|u\|^{1 / 2} \cdot\|\nabla u\|^{1 / 2} \quad \text { with } \quad a_{1}=\left((2 \pi)^{-1}+\sqrt{2}\right)^{1 / 2},
$$

we obtain (10).

In a similar way we can establish the estimates

$$
\begin{aligned}
& \left|(J(u, v), w)_{0}\right| \leq c_{0}\|\nabla u\|_{0}^{1 / 2} \cdot\|\Delta u\|_{0}^{1 / 2} \cdot\|\nabla v\|_{0}^{1 / 2} \cdot\|\Delta v\|_{0}^{1 / 2} \cdot\|w\|_{0}, \\
& \left|(J(u, v), w)_{0}\right| \leq c_{0}\|u\|_{0}^{1 / 2} \cdot\|\nabla u\|_{0}^{1 / 2} \cdot\|\nabla v\|_{0}^{1 / 2} \cdot\|\Delta v\|_{0}^{1 / 2} \cdot\|\nabla w\|_{0},
\end{aligned}
$$

which easily imply (11) and (12).

We now transform the two-layer model (11) containing white noise into a system of coupled random partial differential equations. The reason for taking such a transformation is that we need some particular a priori estimates for the trajectories of the system. Often these a priori estimates can be calculated by the Gronwall lemma. For equations containing a white noise there exists no Gronwall lemma. But we can use this technique for the transformed random partial differential equations.

For this purpose we introduce an Ornstein-Uhlenbeck process $\eta(x, y, t, \omega)$ in 
$L_{p e r}^{2}$. This process is defined by the solution of the following linear stochastic partial differential equation

$$
\eta_{t}=\nu(k+1) \Delta \eta+\dot{W},
$$

with periodic boundary condition and with some initial condition $x \in L_{\text {per }}^{2}$ where $k>0$ is a free control parameter and $W$ is a Wiener process in $L_{p e r}^{2}$. We suppose that the covariance operator $Q$ of this Wiener process has a finite trace. As mentioned in Section 2 such a Wiener process generates a metric dynamical system denoted by $(\Omega, \mathcal{F}, \mathbb{P}, \theta)$ where $\theta$ is the Wiener shift introduced in (3). It is well known that this equation has a stationary solution which is generated by a Gaussian random variable $\eta$ in $H_{p e r}^{1}$. In particular, the mapping

$$
t \rightarrow \eta\left(\theta_{t} \omega\right) \in L_{l o c}^{2}\left(-\infty, \infty ; H_{p e r}^{1}\right)
$$

solves this equation. For moments of $\eta$ we obtain:

$$
\mathbb{E}\|\eta\|_{1}^{2} \leq \frac{\operatorname{tr}_{0} Q}{2 \nu(k+1)}, \quad \mathbb{E}\|\eta\|_{1}^{2 n} \leq C_{n}\left(\frac{\operatorname{tr}_{0} Q}{\nu(k+1)}\right)^{n}, n \in \mathbb{N}, C_{n}>0 .
$$

We introduce new variables

$$
\tilde{q}_{1}:=q_{1}-\eta, q_{2}, \tilde{\psi}_{1}:=\psi_{1}+\xi_{1}, \tilde{\psi}_{2}:=\psi_{2}+\xi_{2}
$$

where stationary process $\eta$ solves the problem (13) and $\xi_{1}$ and $\xi_{2}$ are defined such that the elliptic equations (2) remain of the same form

$$
\begin{aligned}
& \tilde{q}_{1}=\Delta \tilde{\psi}_{1}-F_{1} \cdot\left(\tilde{\psi}_{1}-\tilde{\psi}_{2}\right), \\
& q_{2}=\Delta \tilde{\psi}_{2}-F_{2} \cdot\left(\tilde{\psi}_{2}-\tilde{\psi}_{1}\right),
\end{aligned}
$$

The processes $\xi_{1}$ and $\xi_{2}$ are solutions of the linear elliptic equations

$$
\begin{aligned}
& \Delta \xi_{1}-F_{1} \cdot\left(\xi_{1}-\xi_{2}\right)=-\eta, \\
& \Delta \xi_{2}-F_{2} \cdot\left(\xi_{2}-\xi_{1}\right)=0
\end{aligned}
$$

and can be presented in the form

$$
\begin{aligned}
& \xi_{1}=\frac{1}{F_{1}+F_{2}}\left\{F_{2}(-\Delta)^{-1}+F_{1}\left(-\Delta+F_{1}+F_{2}\right)^{-1}\right\} \eta \\
& \xi_{2}=\frac{F_{2}}{F_{1}+F_{2}}\left\{(-\Delta)^{-1}-\left(-\Delta+F_{1}+F_{2}\right)^{-1}\right\} \eta .
\end{aligned}
$$

Thus the processes $\xi_{1}$ and $\xi_{2}$ are smoother in spatial variables than $\eta$. In fact after simple calculations we have the estimates

$$
\left\|\xi_{i}\right\|_{s+2} \leq\|\eta\|_{s}, \quad i=1,2, \quad \text { and } \quad\left\|\xi_{1}-\xi_{2}\right\|_{s+2} \leq\|\eta\|_{s} \quad s \in \mathbb{R} .
$$


Now we obtain the coupled random partial differential equations for new potential vorticities $\tilde{q}_{1}, q_{2}$ :

$$
\begin{aligned}
& \tilde{q}_{1 t}+J\left(\tilde{\psi}_{1}-\xi_{1}, \tilde{q}_{1}+\eta+\beta y\right)=\nu \Delta^{2} \tilde{\psi}_{1}+f \\
& \quad-\nu \Delta^{2} \xi_{1}-\nu(k+1) \Delta \eta, \\
& q_{2 t}+J\left(\tilde{\psi}_{2}-\xi_{2}, q_{2}+\beta y\right)=\nu \Delta^{2} \tilde{\psi}_{2}-r \Delta \tilde{\psi}_{2} \\
& \quad-\nu \Delta^{2} \xi_{2}+r \Delta \xi_{2} .
\end{aligned}
$$

We treat $\eta, \xi_{1}, \xi_{2}$ as known processes. Using (17) we have

$$
\begin{aligned}
-\nu \Delta^{2} \xi_{1}-\nu(k+1) \Delta \eta & =-\nu F_{1}\left(\Delta \xi_{1}-\Delta \xi_{2}\right)-\nu k \Delta \eta, \\
-\nu \Delta^{2} \xi_{2} & =-\nu F_{2}\left(\Delta \xi_{2}-\Delta \xi_{1}\right) .
\end{aligned}
$$

For convenience, we drop the tilde and rewrite the above system. Thus we finally get the coupled system of random partial differential equations

$$
\begin{aligned}
q_{1 t}+J\left(\psi_{1}-\xi_{1}, q_{1}+\eta+\beta y\right)= & \nu \Delta^{2} \psi_{1}+f \\
& -\nu F_{1}\left(\Delta \xi_{1}-\Delta \xi_{2}\right)-\nu k \Delta \eta \\
q_{2 t}+J\left(\psi_{2}-\xi_{2}, q_{2}+\beta y\right)= & \nu \Delta^{2} \psi_{2}-r \Delta \psi_{2} \\
& -\nu F_{2}\left(\Delta \xi_{2}-\Delta \xi_{1}\right)+r \Delta \xi_{2}
\end{aligned}
$$

with

$$
\begin{aligned}
& q_{1}=\Delta \psi_{1}-F_{1} \cdot\left(\psi_{1}-\psi_{2}\right), \\
& q_{2}=\Delta \psi_{2}-F_{2} \cdot\left(\psi_{2}-\psi_{1}\right),
\end{aligned}
$$

in the class of $L$-periodic functions with initial data $q(x, y, 0)=q_{0}(x, y) \equiv$ $\left(q_{01}(x, y), q_{02}(x, y)\right) \in \mathbf{H}_{\text {per }}^{-1}$, where $\eta$ is the stationary solution to (13) and $\xi_{1}$ and $\xi_{2}$ are solves (17) in $H_{p e r}^{2}$.

For the rest of the paper, we work on this coupled system of random partial differential equations for stochastically forced two-layer quasigeostrophic fluid system.

The coefficients of coupled system (19), (20) have similar properties as the coefficients of the corresponding deterministic two-layer quasigeostrophic system (cf. [6], where $N$-layer model with other boundary conditions is considered). Therefore, similar to [6], using the Galerkin method and the compactness argument we can prove the following assertion on well-posedness of problem (19), (20) in the class of $L$-periodic functions.

Theorem 3.2. Let $q_{0} \in \mathbf{H}_{\text {per }}^{-1}$ and $f \in L_{\text {per }}^{2}$. Then for all $\omega \in \Omega$ and for all $T>0$, the system (19), (20) has a unique solution $\{q(t), \psi(t)\}$ such that

$$
q \in C\left([0, T] ; \mathbf{H}_{p e r}^{-1}\right) \cap L^{2}\left(0, T ; \mathbf{L}_{p e r}^{2}\right) \cap L_{l o c}^{2}\left(0, T ; \mathbf{H}_{p e r}^{1}\right) .
$$

The function $\psi$ associated to $q$ by (20) satisfies

$$
\psi \in C\left([0, T] ; \mathbf{H}_{p e r}^{1}\right) \cap L^{2}\left(0, T ; \mathbf{H}_{p e r}^{2}\right) \cap L_{l o c}^{2}\left(0, T ; \mathbf{H}_{p e r}^{3}\right) .
$$

The solution depends continuously on the initial condition $q_{0} \in \mathbf{H}_{\text {per }}^{-1}$. 
By the uniqueness assertion of the last Theorem the solution $t \rightarrow q(t)$ generates a random dynamical system $\varphi$ with continuous $\mathbf{H}_{p e r}^{-1} \ni q \rightarrow \varphi(t, \omega, q)$ on the phase space $\mathbf{H}_{\text {per }}^{-1}$.

\section{Dissipativity of THE RANDOM DYNAMICAL SYSTEM}

Dynamical systems generated by many nonlinear parabolic differential equations have the dissipative property which means that there exists a compact set absorbing the states of the system in finite time. Usually one can choose such a set which is also forward invariant.

We now construct an absorbing forward invariant set for the random dynamical system generated by (19). This set will be a random set.

Theorem 4.1. There exists a compact random set $B(\omega) \subset \mathbf{H}_{\text {per }}^{-1}$ such that

$$
\begin{aligned}
& \varphi(t, \omega, B(\omega)) \subset B\left(\theta_{t} \omega\right) \quad \text { for } t \geq 0, \\
& \varphi(t, \omega, q(\omega)) \subset B\left(\theta_{t} \omega\right) \quad \text { for } t \geq t_{0}(\omega, q)
\end{aligned}
$$

where $q$ is a random variable with values in $\mathbf{H}_{\text {per }}^{-1}$.

We now divide the proof of this theorem in some lemmata. We start with the following:

Lemma 4.2. Let $q(t)$ be the solution of (19). Then $q(t)$ satisfies the following inequality

$$
\begin{aligned}
\frac{d}{d t}\|q(t)\|_{*}^{2} & +\nu\left(h_{1}\left\|\Delta \psi_{1}(t)\right\|_{0}^{2}+h_{2}\left\|\Delta \psi_{2}(t)\right\|_{0}^{2}\right) \\
& \leq d_{0} \cdot\left\|\eta\left(\theta_{t} \omega\right)\right\|_{0}^{2} \cdot\left(h_{1}\left\|\nabla \psi_{1}\right\|_{0}^{2}+h_{2}\left\|\nabla \psi_{2}\right\|_{0}^{2}\right)+m\left(\theta_{t} \omega\right)
\end{aligned}
$$

where

$$
m(\omega)=d_{1}\|\eta(\omega)\|_{0}^{4}+d_{2}\|\eta(\omega)\|_{0}^{2}+d_{3}
$$

and

$$
\begin{aligned}
& d_{0}=\frac{6 c_{0}^{2}}{\nu}\left(1+\frac{p^{2} \nu}{\lambda_{1}^{2} \min \left\{h_{1}, h_{2}\right\}}\right), \\
& d_{1}=\frac{6 c_{0}^{2} h_{1}}{\nu \lambda_{1}} \\
& d_{2}=9\left(\frac{\beta^{2}\left(h_{1}+h_{2}\right)}{\nu \lambda_{1}^{3}}+\frac{\nu p^{2}}{\lambda_{1}^{2}}\left(\frac{1}{h_{1}}+\frac{1}{5 h_{2}}\right)+\frac{r h_{2}}{18 \lambda_{1}}+k^{2} \nu h_{1}\right), \\
& d_{3}=\frac{9 h_{1}}{\nu \lambda_{1}}\|f\|_{-1}^{2} .
\end{aligned}
$$


Proof. Multiplying the equations (19) by $-2 h_{1} \psi_{1},-2 h_{2} \psi_{2}$, respectively, and then adding together, we obtain

$$
\begin{aligned}
\frac{d}{d t}\|q(t)\|_{*}^{2}+ & 2 \nu\left(h_{1}\left\|\Delta \psi_{1}\right\|_{0}^{2}+h_{2}\left\|\Delta \psi_{2}\right\|_{0}^{2}\right)+2 r h_{2}\left\|\nabla \psi_{2}\right\|_{0}^{2} \\
= & 2 h_{1}\left(J\left(\psi_{1}-\xi_{1}, q_{1}+\eta+\beta y\right), \psi_{1}\right)_{0} \\
& +2 h_{2}\left(J\left(\psi_{2}-\xi_{2}, q_{2}+\beta y\right), \psi_{2}\right)_{0}-2 h_{1}\left(f, \psi_{1}\right)_{0}+2 h_{1} \nu k\left(\Delta \eta, \psi_{1}\right)_{0} \\
& +2 \nu p\left(\Delta \xi_{1}-\Delta \xi_{2}, \psi_{1}\right)_{0}+2 \nu p\left(\Delta \xi_{2}-\Delta \xi_{1}, \psi_{2}\right)_{0} \\
& -2 r h_{2}\left(\Delta \xi_{2}, \psi_{2}\right)_{0} .
\end{aligned}
$$

We have by Lemma 3.1

$$
\left(J\left(\psi_{1}, q_{1}+\eta\right), \psi_{1}\right)_{0}=0,\left(J\left(\psi_{2}, q_{2}\right), \psi_{2}\right)_{0}=0 .
$$

A simple calculation shows that $\left(J\left(\psi_{i}, \beta y\right), \psi_{i}\right)_{0}=0$. We now estimate the right hand side terms. On account of (20), (10), (12), the bilinearity of $J$ and (18) we can estimate

$$
\begin{gathered}
-2 \sum_{i=1}^{2} h_{i}\left(J\left(\xi_{i}, q_{i}\right), \psi_{i}\right)_{0}=-2 \sum_{i=1}^{2} h_{i}\left(J\left(\xi_{i}, \Delta \psi_{i}\right), \psi_{i}\right)_{0}+2 p\left(J\left(\xi_{1}-\xi_{2}, \psi_{2}\right), \psi_{1}\right)_{0} \\
\leq 2 c_{0} \sum_{i=1}^{2} h_{i}\left\|\Delta \xi_{i}\right\|_{0}\left\|\Delta \psi_{i}\right\|_{0}\left\|\nabla \psi_{i}\right\|_{0}+2 c_{1} p\left\|\Delta\left(\xi_{1}-\xi_{2}\right)\right\|_{0}\left\|\nabla \psi_{1}\right\|_{0}\left\|\nabla \psi_{2}\right\|_{0} \\
\leq \frac{\nu}{6}\left(h_{1}\left\|\Delta \psi_{1}\right\|_{0}^{2}+h_{2}\left\|\Delta \psi_{2}\right\|_{0}^{2}\right)+6 \frac{c_{0}^{2}}{\nu}\|\eta\|_{0}^{2}\left(h_{1}\left\|\nabla \psi_{1}\right\|_{0}^{2}+h_{2}\left\|\nabla \psi_{2}\right\|_{0}^{2}\right) \\
\quad+\frac{c_{1} p\|\eta\|_{0}}{\min \left\{h_{1}, h_{2}\right\}}\left(h_{1}\left\|\nabla \psi_{1}\right\|_{0}^{2}+h_{2}\left\|\nabla \psi_{2}\right\|_{0}^{2}\right) \\
\leq \frac{\nu}{3}\left(h_{1}\left\|\Delta \psi_{1}\right\|_{0}^{2}+h_{2}\left\|\Delta \psi_{2}\right\|_{0}^{2}\right)+d_{0}\|\eta\|_{0}^{2}\left(h_{1}\left\|\nabla \psi_{1}\right\|_{0}^{2}+h_{2}\left\|\nabla \psi_{2}\right\|_{0}^{2}\right) .
\end{gathered}
$$

Similarly, due to (11) and (18)

$$
-2 h_{1}\left(J\left(\xi_{1}, \eta\right), \psi_{1}\right)_{0} \leq 2 c_{1} h_{1}\left\|\Delta \xi_{1}\right\|_{0}\|\eta\|_{0}\left\|\Delta \psi_{1}\right\|_{0} \leq \frac{6 c_{1}^{2} h_{1}\|\eta\|_{0}^{4}}{\nu}+\frac{\nu h_{1}}{6}\left\|\Delta \psi_{1}\right\|_{0}^{2} .
$$

By the Cauchy-Schwarz inequality

$$
\begin{aligned}
& -2 \sum_{i=1}^{2} h_{i}\left(J\left(\xi_{i}, \beta y\right), \psi_{i}\right)_{0} \leq 2 \beta \sum_{i=1}^{2} h_{i}\left\|\nabla \xi_{i}\right\|_{-1}\left\|\nabla \psi_{i}\right\|_{0} \\
& \leq \frac{9 \beta^{2}\left(h_{1}+h_{2}\right)}{\nu \lambda_{1}^{3}}\|\eta\|_{0}^{2}+\frac{\nu}{9}\left(h_{1}\left\|\Delta \psi_{1}\right\|_{0}^{2}+h_{2}\left\|\Delta \psi_{2}\right\|_{0}^{2}\right), \\
& -2 h_{1}\left(f, \psi_{1}\right)_{0} \leq 2 h_{1}\|f\|_{-1}\left\|\nabla \psi_{1}\right\|_{0} \leq \frac{9 h_{1}}{\nu \lambda_{1}}\|f\|_{-1}^{2}+\frac{\nu h_{1}}{9}\left\|\Delta \psi_{1}\right\|_{0}^{2} \\
& +2 h_{1} \nu\left(k \Delta \eta, \psi_{1}\right)_{0} \leq 9 k^{2} \nu h_{1}\|\eta\|_{0}^{2}+\frac{\nu h_{1}}{9}\left\|\Delta \psi_{1}\right\|_{0}^{2} .
\end{aligned}
$$


Using (18) once more

$$
\begin{aligned}
2 \nu p\left(\Delta\left(\xi_{1}-\xi_{2}\right), \psi_{1}\right)_{0} & \leq 2 \nu p\left\|\Delta \xi_{1}-\Delta \xi_{2}\right\|_{-2}\left\|\Delta \psi_{1}\right\|_{0} \\
& \leq \frac{9 \nu p^{2}}{\lambda_{1}^{2} h_{1}}\|\eta\|_{0}^{2}+\frac{\nu h_{1}}{9}\left\|\Delta \psi_{1}\right\|_{0}^{2}, \\
-2 \nu p\left(\Delta\left(\xi_{1}-\xi_{2}\right), \psi_{2}\right)_{0} & \leq \frac{9 \nu p^{2}}{5 \lambda_{1}^{2} h_{2}}\|\eta\|_{0}^{2}+\frac{5 \nu h_{2}}{9}\left\|\Delta \psi_{2}\right\|_{0}^{2}, \\
-2 r h_{2}\left(\Delta \xi_{2}, \psi_{2}\right)_{0} & \leq \frac{r h_{2}}{2 \lambda_{1}}\|\eta\|_{0}^{2}+2 r h_{2}\left\|\nabla \psi_{2}\right\|_{0}^{2} .
\end{aligned}
$$

Adding all these inequalities together we obtain the conclusion.

We now consider the random variable $\eta$ defined in Section 2. Recall that $\eta$ depends on the control parameter $k$. If $k$ is chosen large enough then particular moments of $\eta$ are small. Especially we can formulate:

Lemma 4.3. Let $W$ be a Wiener process in $L_{\text {per }}^{2}$ with finite trace of the covariance. Then under assumptions

$$
\frac{2 d_{0} a_{0} \operatorname{tr}_{0} Q}{\lambda_{1}^{2} \nu^{2}(k+1)}<1, \quad \frac{16 d_{0} \operatorname{tr}_{0} Q}{\lambda_{1}^{2} \nu^{2}(k+1)^{2}}<1
$$

the random variable

$$
R_{0}(\omega):=\int_{-\infty}^{0} e^{\frac{\nu \lambda \tau}{a_{0}} \tau+d_{0} \int_{\tau}^{0}\left\|\eta\left(\theta_{\tau^{\prime}} \omega\right)\right\|_{0}^{2} d \tau^{\prime}} m\left(\theta_{\tau} \omega\right) d \tau
$$

is finite and tempered. Moreover

$$
\left(\mathbb{E} R_{0}^{2}\right)^{1 / 2} \leq d_{4}^{2}\left(\frac{3 a_{0}}{2 \nu \lambda_{1}}\right)^{3 / 2} \cdot\left(\frac{2 \lambda_{1} \nu}{a_{0}}-\frac{4 d_{0} \operatorname{tr}_{0} Q}{\lambda_{1} \nu(k+1)}\right)^{-1 / 2}
$$

where

$$
d_{4}=C_{8}^{1 / 4} \frac{d_{1}\left(\operatorname{tr}_{0} Q\right)^{2}}{\lambda_{1}^{2} \nu^{2}(k+1)^{2}}+C_{4}^{1 / 4} \frac{d_{2} \operatorname{tr}_{0} Q}{\lambda_{1} \nu(k+1)}+d_{3}
$$

is an estimate for $\left(\mathbb{E} m^{4}\right)^{1 / 4}$ (the constants $C_{8}, C_{4}$ are defined in (14)).

The proof of this lemma can be found in Chueshov et. al. 13 for an Ornstein-Uhlenbeck process in another Hilbert space. However the argument given there is of a general nature.

We now construct a set satisfying (21).

Lemma 4.4. Let $R(\omega):=a R_{0}(\omega)$ for some $a>1$ and $R_{0}$ as in Lemma 4.3. Then the closed $\mathbf{H}_{\text {per }}^{-1}$-ball $B\left(0, R(\omega)^{\frac{1}{2}}\right)$ fullfills (21) provided conditions (29) hold.

Proof. Using Lemma 4.2 and relation (7) we have

$$
\frac{d}{d t}\|q(t)\|_{*}^{2} \leq\left(-\frac{\nu \lambda_{1}}{a_{0}}+d_{0} \cdot\left\|\eta\left(\theta_{t} \omega\right)\right\|_{0}^{2}\right) \cdot\|q(t)\|_{*}^{2}+m\left(\theta_{t} \omega\right) .
$$


Let $q_{0}=q(0)$ and $\rho\left(t, \omega,\left\|q_{0}\right\|_{*}^{2}\right)$ be the solution of one dimensional random affine equation

$$
\frac{d \rho(t)}{d t}+\frac{\nu \lambda_{1}}{a_{0}} \rho=d_{0}\left\|\eta\left(\theta_{t} \omega\right)\right\|_{0}^{2} \rho+m\left(\theta_{t} \omega\right), \quad \rho\left(0, \omega,\left\|q_{0}\right\|_{*}^{2}\right)=\left\|q_{0}\right\|_{*}^{2} .
$$

A comparison argument gives that

$$
\left\|\varphi\left(t, \omega, q_{0}\right)\right\|_{*}^{2} \equiv\|q(t)\|_{*}^{2} \leq \rho\left(t, \omega,\left\|q_{0}\right\|_{*}^{2}\right) .
$$

Here $\varphi$ is the dynamical system introduced in Section 3: $\varphi\left(t, \omega, q_{0}\right)=q(t)$, where $q(t)$ is the solution to (19) with the initial data $q_{0}$. Equation (23) has the stationary solution given by $t \rightarrow R_{0}\left(\theta_{t} \omega\right)$ :

$$
\rho\left(t, \omega, R_{0}(\omega)\right)=R_{0}\left(\theta_{t} \omega\right) .
$$

This can be checked by the variation of constants formula. This solution is exponentially attracting which follows from the variation of constants formula again:

$$
\begin{aligned}
\left|R_{0}\left(\theta_{t} \omega\right)-\rho\left(t, \omega,\left\|q_{0}\right\|_{*}^{2}\right)\right| & =\left|\rho\left(t, \omega, R_{0}(\omega)\right)-\rho\left(t, \omega,\left\|q_{0}\right\|_{*}^{2}\right)\right| \\
& \leq e^{\int_{0}^{t}\left(d_{0}\left\|\eta\left(\theta_{\tau} \omega\right)\right\|_{0}^{2}-\frac{\nu \lambda_{1}}{a_{0}}\right) d \tau}\left(R(\omega)+\left\|q_{0}\right\|_{*}^{2}\right)
\end{aligned}
$$

which tends to zero exponentially fast. Indeed, it follows from (14) and (22) that for a sufficient small $\varepsilon>0$,

$$
\int_{0}^{t} d_{0}\left\|\eta\left(\theta_{\tau} \omega\right)\right\|_{0}^{2} d \tau<\frac{\nu \lambda_{1}-\varepsilon}{a_{0}} t, \quad \frac{\nu \lambda_{1}-\varepsilon}{a_{0}}>0
$$

for large $t>0$ under conditions (22).

It remains to prove the existence of a compact set $B$ satisfying (23).

Lemma 4.5. Suppose that the random variable $R(\omega)$ is defined in Lemma 4.4. The set

$$
B(\omega):=\overline{\varphi\left(1, \theta_{-1} \omega, B\left(0, R\left(\theta_{-1} \omega\right)^{\frac{1}{2}}\right)\right)}
$$

is a compact absorbing forward invariant random set. Moreover

$$
\omega \mapsto \sup \left\{\left\|\Delta \psi_{1}\right\|_{0}^{2}+\left\|\Delta \psi_{1}\right\|_{0}^{2}:\left(q_{1}, q_{2}\right) \in B(\omega)\right\}
$$

is a tempered random variable ( $\psi_{1}$ and $\psi_{2}$ are defined by (20)).

Proof. Since $\varphi(t, \omega, \cdot)$ is completely continuous for $t>0$ (see the regularity assertion of Theorem 3.2) the sets $B(\omega)$ are compact. Since $R$ is a random variable the ball $B\left(0, R^{\frac{1}{2}}\right)$ is a random set. The continuity of $\varphi(t, \omega, \cdot)$ allows us to conclude that $B$ is a random set. The construction of $B$ ensures that that set in absorbing and forward invariant. The temperedness of (24) can be proved in the same way as in 13 for the $2 D$ Navier-Stokes equations.

Hence we have shown that the two-layer quasigeostrophic flow system is dissipative in the probabilistic sense. In the next section we consider asymptotic probabilistic determining functionals. 


\section{Determinging FunCtionals}

In this section, we compare two solutions as a way to understand asymptotic dynamics in the probabilistic sense. Consider the difference of two solutions $\hat{q}$ and $\bar{q}$, corresponding to the stream functions $\hat{\psi}, \bar{\psi}$. We set

$$
q=\left(q_{1}, q_{2}\right)=\hat{q}-\bar{q},
$$

corresponding to the stream function

$$
\psi=\hat{\psi}-\bar{\psi}
$$

We get random partial differential equations for the solution difference $q=$ $\hat{q}-\bar{q}$ from (19):

$$
\begin{aligned}
q_{1 t}= & \nu \Delta^{2} \psi_{1} \\
& -J\left(\psi_{1}, \hat{q}_{1}+\beta y\right)-J\left(\bar{\psi}_{1}, q_{1}\right)-J\left(\psi_{1}, \eta\right)+J\left(\xi_{1}, q_{1}\right), \\
q_{2 t}= & \nu \Delta^{2} \psi_{2}-r \Delta \psi_{2} \\
& -J\left(\psi_{2}, \hat{q}_{2}+\beta y\right)-J\left(\bar{\psi}_{2}, q_{2}\right)+J\left(\xi_{2}, q_{2}\right),
\end{aligned}
$$

Multiplying (25) by $-h_{1} \psi_{1}$, and (26) by $-h_{2} \psi_{2}$ and adding together, we get

$$
\begin{aligned}
\frac{1}{2} \frac{d}{d t}\|q\|_{*}^{2} & =-\nu\left[h_{1}\left\|\Delta \psi_{1}\right\|_{0}^{2}+h_{2}\left\|\Delta \psi_{2}\right\|_{0}^{2}\right]-r h_{2}\left\|\nabla \psi_{2}\right\|_{0}^{2} \\
& +h_{1}\left(J\left(\bar{\psi}_{1}-\xi_{1}, q_{1}\right), \psi_{1}\right)_{0}+h_{2}\left(J\left(\bar{\psi}_{2}-\xi_{2}, q_{2}\right), \psi_{2}\right)_{0} .
\end{aligned}
$$

We start with

$$
h_{1}\left(J\left(\bar{\psi}_{1}-\xi_{1}, q_{1}\right), \psi_{1}\right)_{0} \equiv I_{1}+I_{2}
$$

where

$$
I_{1}=h_{1}\left(J\left(\bar{\psi}_{1}-\xi_{1}, \Delta \psi_{1}\right), \psi_{1}\right)_{0}, \quad I_{2}=p\left(J\left(\bar{\psi}_{1}-\xi_{1}, \psi_{2}\right), \psi_{1}\right)_{0} .
$$

By (10) we have

$$
\left|I_{1}\right| \leq c_{0} h_{1}\left\|\bar{\psi}_{1}-\xi_{1}\right\|_{2}\left\|\Delta \psi_{1}\right\|_{0}\left\|\nabla \psi_{1}\right\|_{0} .
$$

Using (12) we obtain

$$
\left|I_{2}\right| \leq c_{1} p\left\|\bar{\psi}_{1}-\xi_{1}\right\|_{2}\left\|\nabla \psi_{2}\right\|_{0}\left\|\nabla \psi_{1}\right\|_{0}
$$

Thus

$$
\left|I_{2}\right| \leq c_{1} p \lambda_{1}^{-\frac{1}{2}}\left\|\bar{\psi}_{1}-\xi_{1}\right\|_{2}\left\|\Delta \psi_{2}\right\|_{0}\left\|\nabla \psi_{1}\right\|_{0} .
$$

Using the inequality $a b \leq \epsilon a^{2}+\frac{1}{4 \epsilon} b^{2}$, we get

$$
\begin{array}{r}
h_{1}\left(J\left(\bar{\psi}_{1}-\xi_{1}, q_{1}\right), \psi_{1}\right)_{0} \leq \frac{\nu}{4}\left(h_{1}\left\|\Delta \psi_{1}\right\|_{0}^{2}+h_{2}\left\|\Delta \psi_{2}\right\|_{0}^{2}\right) \\
+\frac{h_{1} c_{0}^{2}}{\nu}\left(1+F_{1} F_{2} \lambda_{1}^{-2}\right)\left\|\bar{\psi}_{1}-\xi_{1}\right\|_{2}^{2}\left\|\nabla \psi_{1}\right\|_{0}^{2} .
\end{array}
$$


In a similar way we have

$$
\begin{aligned}
& h_{2}\left(J\left(\bar{\psi}_{2}-\xi_{2}, q_{2}\right), \psi_{2}\right)_{0} \leq \frac{\nu}{4}\left(h_{1}\left\|\Delta \psi_{1}\right\|_{0}^{2}+h_{2}\left\|\Delta \psi_{2}\right\|_{0}^{2}\right) \\
&+\frac{h_{2} c_{0}^{2}}{\nu}\left(1+F_{1} F_{2} \lambda_{1}^{-2}\right)\left\|\bar{\psi}_{2}-\xi_{2}\right\|_{2}^{2}\left\|\nabla \psi_{2}\right\|_{0}^{2} .
\end{aligned}
$$

Using (27), (28) and (29) we obtain

$$
\begin{aligned}
\frac{d}{d t}\|q\|_{*}^{2} \leq & -\nu\left[h_{1}\left\|\Delta \psi_{1}\right\|_{0}^{2}+h_{2}\left\|\Delta \psi_{2}\right\|_{0}^{2}\right]-2 r h_{2}\left\|\nabla \psi_{2}\right\|_{0}^{2} \\
& +b_{0}\left(h_{1}\left\|\bar{\psi}_{1}-\xi_{1}\right\|_{2}^{2}\left\|\nabla \psi_{1}\right\|_{0}^{2}+h_{2}\left\|\bar{\psi}_{2}-\xi_{2}\right\|_{2}^{2}\left\|\nabla \psi_{2}\right\|_{0}^{2}\right),
\end{aligned}
$$

where $b_{0}=\frac{2 c_{0}^{2}}{\nu}\left(1+F_{1} F_{2} \lambda_{1}^{-2}\right)$. This estimate is the main point in the construction of determining functionals. It is our aim to obtain an integral equation like (5). To this end we first consider the case when parameter $r>0$ is arbitrary. Let $\mathcal{L}=\left\{l_{j}\right\}_{1}^{N}$ be a set of linearly independent bounded linear functionals on the space $\mathbf{H}_{\text {per }}^{2}$. Assume that the set $\mathcal{L}$ possesses the property

$$
\|\psi\|_{1} \leq C_{\mathcal{L}} \max _{j}\left|l_{j}(\psi)\right|+\varepsilon_{\mathcal{L}}\|\psi\|_{2}
$$

for any $\psi \in \mathbf{H}_{\text {per }}^{2}$ with some positive constant $C_{\mathcal{L}}$ and $\varepsilon_{\mathcal{L}}$. We note that the best possible value of the parameter $\varepsilon_{\mathcal{L}}$ is called the completeness defect of the family $\mathcal{L}$ with respect of the pair of the spaces $\mathbf{H}_{\text {per }}^{1}$ and $\mathbf{H}_{\text {per }}^{2}$ (see [10, 11]) and the smallness of the parameter $\varepsilon_{\mathcal{L}}$ is crucial in the subsequent considerations. We refer to [10, 11] for general properties of completeness defect and for estimates of $\varepsilon_{\mathcal{L}}$ for several families of functionals on Sobolev spaces.

From (31) for $\psi=\left(\sqrt{h_{1}} \psi_{1}, \sqrt{h_{2}} \psi_{2}\right)$ we have

$$
\begin{aligned}
\left(h_{1}\left\|\Delta \psi_{1}\right\|_{0}^{2}+h_{2}\left\|\Delta \psi_{2}\right\|_{0}^{2}\right) \geq & \frac{1-\delta}{\varepsilon_{\mathcal{L}}^{2}}\left(h_{1}\left\|\nabla \psi_{1}\right\|_{0}^{2}+\left\|h_{2} \nabla \psi_{2}\right\|_{0}^{2}\right) \\
& -C_{\delta, \mathcal{L}} \max _{j=1, \cdots, N}\left|l_{j}\left(\sqrt{h_{1}} \psi_{1}, \sqrt{h_{2}} \psi_{2}\right)\right|^{2} .
\end{aligned}
$$

for any $0<\delta<1$ with appropriate positive constant $C_{\delta, \mathcal{L}}$. Therefore from (30) and (7) we obtain

$$
\frac{d}{d t}\|q\|_{*}^{2} \leq l_{\mathcal{L}}^{(1)}(\bar{\psi}, \omega)\|q\|_{*}^{2}+C_{\delta, \mathcal{L}} \max _{j=1, \cdots, N}\left|l_{j}\left(\sqrt{h_{1}} \psi_{1}, \sqrt{h_{2}} \psi_{2}\right)\right|^{2},
$$

where

$$
l_{\mathcal{L}}^{(1)}(\bar{\psi}, \omega)=-\frac{1-\delta}{a_{0} \varepsilon_{\mathcal{L}}^{2}}+b_{0} \max \left\{\left\|\bar{\psi}_{1}-\xi_{1}\right\|_{2}^{2},\left\|\bar{\psi}_{2}-\xi_{2}\right\|_{2}^{2}\right\} .
$$

The application of Theorem 2.2 gives the following assertion.

Theorem 5.1. Let $\mathcal{L}=\left\{l_{j}: j=1, \cdots, N\right\}$ be a finite set of linearly independent continuous functionals on the space $\mathbf{H}_{\text {per }}^{2}$. We assume that this set 
satisfies (31). Let (22) hold. If

$$
\varepsilon_{\mathcal{L}}<\frac{\nu \sqrt{\min \left\{h_{1}, h_{2}\right\}}}{\sqrt{2 a_{0} b_{0} \Sigma}},
$$

where

$$
\Sigma=d_{0}\left(\mathbb{E}\|\eta\|_{0}^{4}\right)^{\frac{1}{2}}\left(\mathbb{E} R^{2}\right)^{\frac{1}{2}}+\mathbb{E} m+\nu \min \left\{h_{1}, h_{2}\right\} \mathbb{E}\|\eta\|_{0}^{2}
$$

and $m$, involving the Wiener process through the Ornstein-Uhlenbeck process $\eta$, is defined in Lemma 4.9, then $\mathcal{L}^{h_{1}, h_{2}}=\left\{l_{j}^{h_{1}, h_{2}}: j=1, \cdots, N\right\}$ where $l_{j}^{h_{1}, h_{2}}\left(\psi_{1}, \psi_{2}\right):=l_{j}\left(\sqrt{h_{1}} \psi_{1}, \sqrt{h_{2}} \psi_{2}\right), l_{j} \in \mathcal{L}$ is a set of asymptotically determining functionals in probability for the stochastically forced two-layer quasigeostrophic fluid system (19), (20).

Proof. We integrate (32). The conditions of Theorem (2.2) are satisfied if $\mathbb{E} l_{\mathcal{L}^{(1)}}<0 . l_{\mathcal{L}^{(1)}}$ can be estimated by

$$
-\nu \frac{1-\delta}{a_{0} \varepsilon_{\mathcal{L}}^{2}}+\frac{2 \nu b_{0}}{\nu \min \left\{h_{1}, h_{2}\right\}}\left(h_{1}\left\|\Delta \bar{\psi}_{1}\right\|_{0}^{2}+h_{2}\left\|\Delta \bar{\psi}_{2}\right\|_{0}^{2}\right)+2 b_{0}\|\eta\|_{0}^{2} .
$$

On account of Lemma 4.2

$$
\sup _{x \in B(\omega)} \frac{1}{t} \nu \int_{0}^{t}\left(h_{1}\left\|\Delta \bar{\psi}_{1}(\tau, \omega, x)\right\|_{0}^{2}+h_{1}\left\|\Delta \bar{\psi}_{2}(\tau, \omega, x)\right\|_{0}^{2}\right) d \tau
$$

has the bound

$$
\frac{1}{t}\left(R(\omega)+\int_{0}^{t}\left(d_{0}\left\|\eta\left(\theta_{\tau} \omega\right)\right\|_{0}^{2} R\left(\theta_{\tau} \omega\right)+m\left(\theta_{\tau} \omega\right)\right) d \tau\right)
$$

since $B$ is forward invariant. Note that the expectation of $R / t$ can be made arbitrarily small if $t$ is large. Calculation the expectation of the last expression and choosing $t$ sufficiently large yields the conclusion.

Remark 5.2. i) It is easily seen that there exists a set $\hat{\mathcal{L}}^{h_{1}, h_{2}}$ of linearly independent linear bounded functionals $\left\{\hat{l}_{j}\right\}_{1}^{N}$ which are determining with respect to $q$. Indeed, $q$ and $\psi$ are connected by a linear homeomorphism $\Lambda$ from $\mathbf{L}_{\text {per }}^{2}$ to $\mathbf{H}_{\text {per }}^{2}$ defined by (20) such that we can set $l_{j}^{h_{1}, h_{2}}=l_{j}^{h_{1}, h_{2}} \circ \Lambda$ where $\hat{\mathcal{L}}=\left\{l_{j}\right\}_{1}^{N}$ defines the set of determining functionals introduced in Theorem 5.1.

ii) If $h_{1}=h_{2}$ then the set $\mathcal{L}$ itself is determining in the sense of Definition 2.1.

iii) Assumption (22) holds, if

$$
\frac{4 d_{0} a_{0} \operatorname{tr}_{0} Q}{\lambda_{1}^{2} \nu^{2}(k+1)}<1, \quad k+1>\frac{4}{a_{0}}
$$


for example. In this case it is easy to see that $\left(\mathbb{E} R_{0}^{2}\right)^{1 / 2} \leq 4 d_{4}^{2}\left(\frac{3 a_{0}}{2 \nu \lambda_{1}}\right)^{2}$ (we choose $a=4 / 3$ in Lemma 4.4). Therefore using (14) we obtain

$$
\Sigma \leq C_{4}^{1 / 2} \frac{\operatorname{tr}_{0} Q}{\nu(k+1)} \cdot\left(4 d_{0} d_{4}^{2}\left(\frac{3 a_{0}}{2 \nu \lambda_{1}}\right)^{2}+\nu \min \left\{h_{1}, h_{2}\right\}\right)+d_{4} .
$$

Using (34) again we have the estimate

$$
d_{4} \leq \frac{\operatorname{tr}_{0} Q}{k+1} \cdot\left(C_{8}^{1 / 4} \frac{d_{1}}{4 d_{0} a_{0}}+C_{4}^{1 / 4} \frac{d_{2}}{\lambda_{1} \nu}\right)+d_{3} .
$$

Therefore under conditions (34) in the deterministic limit $\operatorname{tr}_{0} Q \rightarrow 0$ we obtain estimate

(35) $\varepsilon_{\mathcal{L}}<\frac{\nu^{2} \lambda_{1} \sqrt{\min \left\{h_{1}, h_{2}\right\}}}{6\|f\|_{-1} c_{0} \sqrt{h_{1}}} \cdot\left\{\left(\lambda_{1}+2 \max \left\{F_{1}, F_{2}\right\}\right)\left(1+\frac{F_{1} F_{2}}{\lambda_{1}^{2}}\right)\right\}^{-1 / 2}$.

If this estimate holds, then functionals $\left\{l_{j}^{h_{1}, h_{2}}\right\}$ are determining for the deterministic two-layer model. Moreover under condition (35) these functionals are also determining for our stochastic two-layer model (11) and (2) provided the noise parameter $\operatorname{tr}_{0} Q$ is small enough.

At the end of this section, we will discuss appropriate values for fluid parameters. We will estimate the sufficient condition in Theorem 5.1, namely the inequality (35), in terms of fluid parameters.

We further show that the long-time dynamics of the two-layer geophsysical fluid system is determined by the long time dynamics of the top layer alone, when the fluid parameters and the Wiener process satisfy certain conditons. In this case the long time dynamics will also be determined by only finitely many functionals.

Let $\mathcal{L}=\left\{l_{j}: j=1, \ldots, N\right\}$ be a finite set of linearly independent continuous functionals on the space $H_{p e r}^{2}$ of the top stream functions. We assume that

$$
\left\|\nabla \psi_{1}\right\|_{0} \leq \varepsilon_{\mathcal{L}} \cdot\left\|\Delta \psi_{1}\right\|_{0}+C_{\mathcal{L}} \cdot \max _{j=1, \ldots, N}\left|l_{j}\left(\psi_{1}\right)\right|, \quad \psi_{1} \in H_{p e r}^{2}
$$

where $C_{\mathcal{L}}>0$ is a constant depending on $\mathcal{L}$. As above we have

$$
h_{1}\left\|\Delta \psi_{1}\right\|_{0}^{2} \geq \frac{1-\delta}{\varepsilon_{\mathcal{L}}^{2}} h_{1}\left\|\nabla \psi_{1}\right\|_{0}^{2}-C_{\delta, \mathcal{L}} h_{1} \max _{j=1, \cdots, N}\left|l_{j}\left(\psi_{1}\right)\right|^{2} .
$$

for any $0<\delta<1$ with appropriate positive constant $C_{\delta, \mathcal{L}}$.

Thus under the condition (36) and using the inequality $\left\|\nabla \psi_{2}\right\|_{0}^{2} \leq \lambda_{1}\left\|\Delta \psi_{2}\right\|_{0}^{2}$, the estimate (30) for the solution difference $\hat{q}-\bar{q}$ becomes

$$
\begin{aligned}
\frac{d}{d t}\|q\|_{*}^{2} & +\nu h_{1} \frac{1-\delta}{\varepsilon_{\mathcal{L}}^{2}}\left\|\nabla \psi_{1}\right\|_{0}^{2}+\left(\nu \lambda_{1}+2 r\right) h_{2}\left\|\nabla \psi_{2}\right\|_{0}^{2} \\
& \leq b_{0} \max \left\{\left\|\bar{\psi}_{1}-\xi_{1}\right\|_{2}^{2},\left\|\bar{\psi}_{2}-\xi_{2}\right\|_{2}^{2}\right\}\|q\|_{*}^{2}+C_{\delta, \mathcal{L}} \max _{j=1, \cdots, N}\left|l_{j}\left(h_{1} \psi_{1}\right)\right|^{2} .
\end{aligned}
$$


Thus we obtain

$$
\frac{d}{d t}\|q\|_{*}^{2} \leq l_{\mathcal{L}}^{(2)}(\bar{\psi}, \omega)\|q\|_{*}^{2}+C_{\delta, \mathcal{L}} \max _{j=1, \cdots, N}\left|l_{j}\left(h_{1} \psi_{1}\right)\right|^{2},
$$

where

$$
\begin{aligned}
l_{\mathcal{L}}^{(2)}(\bar{\psi}, \omega)= & -\min \left\{\nu \frac{1-\delta}{a_{0} \varepsilon_{\mathcal{L}}^{2}}, \frac{\nu \lambda_{1}+2 r}{a_{0}}\right\} \\
& +b_{0} \max \left\{\left\|\bar{\psi}_{1}-\xi_{1}\right\|_{2}^{2},\left\|\bar{\psi}_{2}-\xi_{2}\right\|_{2}^{2}\right\} .
\end{aligned}
$$

Again applying Theorem 2.2 we obtain the main result.

Theorem 5.3. Let $\mathcal{L}=\left\{l_{j}: j=1, \ldots, N\right\}$ be a finite set of linearly independent continuous functionals on the space $H_{\text {per }}^{2}$. We assume that this set satisfies the following condition involving only with the top fluid layer dynamical variable, i.e., the stream function $\psi_{1}(x, y, t)$

$$
\left\|\nabla \psi_{1}\right\|_{0} \leq \varepsilon_{\mathcal{L}} \cdot\left\|\Delta \psi_{1}\right\|_{0}+C_{\mathcal{L}} \cdot \max _{j=1, \ldots, N}\left|l_{j}\left(\psi_{1}\right)\right|, \quad \psi_{1} \in H_{p e r}^{2}
$$

If

$$
\Sigma<\min \left\{\frac{\nu}{\varepsilon_{\mathcal{L}}^{2}},\left(\nu \lambda_{1}+2 r\right)\right\} \frac{\nu \min \left\{h_{1}, h_{2}\right\}}{2 a_{0} b_{0}},
$$

where $\Sigma$ is given by (33), then $\mathcal{L}$ is a set of asymptotically determining functionals in probability for the stochastically forced two-layer quasigeostrophic fluid system (19), (20).

The proof of this theorem is the same as the proof of Theorem 5.1.

Relation (38) holds if

$$
\frac{\nu}{\varepsilon_{\mathcal{L}}^{2}} \geq \nu \lambda_{1}+2 r
$$

and

$$
\Sigma<\left(\nu \lambda_{1}+2 r\right) \frac{\nu \min \left\{h_{1}, h_{2}\right\}}{2 b_{0} a_{0}} .
$$

The parameter $\Sigma$ depends on $r$ via $d_{2}$. Therefore it is not clear whether (40) holds for some $r$. However as in Remark 5.2 in the deterministic limit $\operatorname{tr}_{0} Q \rightarrow 0$ the estimate (40) turns into the relation

$$
\nu \lambda_{1}+2 r>\frac{36 c_{0}^{2}\|f\|_{-1}^{2} h_{1}}{\nu^{3} \lambda_{1} \min \left\{h_{1}, h_{2}\right\}} \cdot\left(1+\frac{2}{\lambda_{1}} \max \left\{F_{1}, F_{2}\right\}\right)\left(1+\frac{F_{1} F_{2}}{\lambda_{1}^{2}}\right) .
$$

This observation leads to the following assertion.

Corollary 5.4. Assume that (41) holds. Let $\mathcal{L}=\left\{l_{j}: j=1, \ldots, N\right\}$ be a finite set of linearly independent continuous functionals on the space $H_{p e r}^{2}$. We assume that this set satisfies (37) with the parameter $\varepsilon_{\mathcal{L}}$ satisfying (39). 
Then there exists $\gamma>0$ such that $\mathcal{L}$ is a set of asymptotically determining functionals in probability for the stochastically forced two-layer quasigeostrophic fluid system (19), (20) provided $\operatorname{tr}_{0} Q \leq \gamma$.

Consequencely, the asymptotic probabilistic dynamics of the stochastically forced two-layer quasigeostrophic fluid system is determined only by the top fluid layer.

Remark 5.5. Note that the main task is to prove the existence of determining functionals of the main equation (11). It is easily seen by the structure the transformations (15) a set $\mathcal{L}$ is determining in probability for $(19)$ if and only if the same set $\mathcal{L}$ is determining in probability for (11); see [13].

As Theorems 5.1 and 5.3 show the problem of describing of finite families of determining functionals is reduced to the study of sets of functionals for which the estimate (31) (or (36)) holds with $\varepsilon_{\mathcal{L}}$ small enough. It is also important to calculate the best possible value for $\varepsilon_{\mathcal{L}}$ for the given family of functional. For this parameter there is the estimate from below depending only on a number of functionals [10] and this estimate coinside with $\varepsilon_{\mathcal{L}}$ when functionals are modes (see Example 1 below). We refer to [10, 11] for further discussion concerning an optimal choice of families of functionals with properties like (31) or (36). We also note that the deterministic counterparts of Theorems 5.1 and 5.3 were proved in [7] for other boundary conditions.

Example 1 (Determining modes) Let $\left\{e_{i}(x, y)\right\}_{i=1}^{\infty}$ be the basis of eigenfunctions of $-\Delta$ with the periodic boundary conditions in $O$ such that the corresponding eigenvalues possesses the property

$$
0<\lambda_{1} \leq \lambda_{2} \leq \ldots
$$

We note that every eigenfunction has the form $L^{-1} \exp \left\{\frac{2 \pi}{L}\left(j_{1} x+j_{2} y\right)\right\}$. However we numerate them such that (42) holds. Suppose $\mathcal{L}_{N}=\left\{l_{j}\right.$ : $j=1, \ldots, N\}$ is the set of the functionals on $H_{p e r}^{2}$ of the form

$$
l_{j}(u)=\int_{O} u(x, y) e_{j}(x, y) d O, \quad j=1, \ldots, N .
$$

Then one can prove (see, e.g. [10]) that estimate (36) holds for $\mathcal{L}_{N}$ with $\varepsilon_{\mathcal{L}_{N}}=\lambda_{N+1}^{-1 / 2}$ and this value is the best possible among all families of functionals consisting of $N$ elements. Since $\lambda_{N} \sim c_{0} N L^{-2}$ for $N$ large enough, we have that $\varepsilon_{\mathcal{L}_{N}} \sim \tilde{c}_{0} L / \sqrt{N}$ with some absolute constant $\tilde{c}_{0}$. Therefore we can use Theorem 5.3 to estimate number of determining modes. We also note that using the family $\mathcal{L}_{N}$ of the functionals on $H_{\text {per }}^{2}$ we can easily construct a family $\tilde{\mathcal{L}}_{N}$ of the functionals on $\mathbf{H}_{\text {per }}^{2}=H_{\text {per }}^{2} \times H_{\text {per }}^{2}$ such that (31) holds with $\varepsilon_{\mathcal{L}_{N}}=\lambda_{N+1}^{-1 / 2}$.

Example 2 (Determining nodes) Let us consider the nodes

$$
\left(x_{i}, y_{j}\right)=\frac{L}{\sqrt{N}} \cdot(i, j) \in O, \quad 1 \leq i, j \leq \sqrt{N}
$$


and define functionals $l_{i j}$ on $H_{\text {per }}^{2}$ as $\delta$-functions at $\left(x_{i}, y_{j}\right)$, i.e by the formulas $l_{i j}(u)=u\left(x_{i}, y_{j}\right)$. Let $\mathcal{L}=\left\{l_{i j}\right\}$. One can prove (see, e.g. 10] or [25]) that estimate (36) holds for $\mathcal{L}$ with $\varepsilon_{\mathcal{L}}=\bar{c}_{0} L / \sqrt{N}$ where $\bar{c}_{0}=\frac{1}{2}$ is an absolute constant [25]. Thus $\varepsilon_{\mathcal{L}}$ has the same order for large $N$ as is the best possible value $\varepsilon_{\mathcal{L}_{N}}$ for families of functionals consisting of $N$ elements.

We also note that the estimates for the completeness defect $\varepsilon_{\mathcal{L}}$ and the Ekman constant $r$ given in Theorems 5.1 and 5.3 are rather crude. In the two-layer quasigeostrophic model considered above, the parameter $\nu$ is the molecular viscosity. For ocean water, $\nu=10^{-6} \mathrm{~m}^{2} \mathrm{~s}^{-1}$. However, fluid turbulence at small scales can act as an extra dissipative mechanism, thus calling for the substitution of the molecular viscosity by a much larger eddy viscosity. For example, in the two-layer quasigeostrophic model flow simulation in [28], the eddy viscosity (we still use the same notation as the molecular viscosity) is taken as $\nu=50 \mathrm{~m}^{2} \mathrm{~s}^{-1}$. The gravitational acceleration $g=9.81 \mathrm{~ms}^{-2}$.

At mid-latitude $(45$ degree $N), f_{0}=8 \times 10^{-5} s^{-1}$ and $\beta=2.3 \times 10^{-11} \mathrm{~m}^{-1} \mathrm{~s}^{-1}$. For large-scale flows at mid-latitude, such as the Gulf Stream in the Atlantic ocean, the horizontal spatial scale $L$ is at the order of $1000 \mathrm{~km}$. Moreover, layer depth $h_{1}$ and $h_{2}$ are at the order of $500 \mathrm{~m}$ each for large scale flows such as the Gulf Stream at the mid-latitude in the Atlantic ocean. For the eddy viscosity $50 \mathrm{~m}^{2} \mathrm{~s}^{-1}$ in [28], the Ekman constant $r$ is then at the order of $10^{-5} s^{-1}$. The number $r$ is large when the eddy viscosity is taken to be large.

The ocean water mean density $\rho_{0}$ is about $1025 \mathrm{kgm}^{-3}$ or $1.025 \mathrm{gcm}^{-3}$. $\rho_{1}$ and $\rho_{2}$ are in the vicinity of $\rho_{0}$. The density difference $\rho_{2}-\rho_{1} \approx 25 \mathrm{kgm}^{-3}$. But it is this small density difference that in turn determines pressure differences and thereby drive the ocean circulation [34].

The mean wind forcing $f$, i.e., the deterministic part of the curl of the wind stree on the top fluid layer is usually taken as a stationary, or being timeaveraged and even also zonally averaged, sinusoidal function. For example [16],

$$
f=\frac{2 \pi \tau_{0}}{\rho_{0} h_{1} L} \sin \frac{2 \pi y}{L}
$$

where the wind tension $\tau_{0}$ is of order 1 dyne $/ \mathrm{cm}^{2}$ or of order $0.1 \mathrm{~N} / \mathrm{m}^{2}$. With this mean wind forcing and physical parameters specified above, the inequality (35) turns into the estimate $\varepsilon_{\mathcal{L}} \leq 1.35 \cdot 10^{-3} \mathrm{~m}$ and therefore in the case of Examples 1 or 2 for the number $N$ of functionals we obtain the estimate $N \approx 10^{18}$. Thus Theorem 5.1 should be only considered as an qualitative assertion about finite-dimensionality of the long-time behaviour of the stochastically forced two-layer quasigeostrophic fluid system. As for Theorem 5.3, a similar calculations show that the condition (41) can be valid under some special choice of parameters. Therefore Theorem 5.3 only 
predicts the possibility of a situation when the bottom fluid layer is slaved by the top layer.

\section{Summary}

We have considered asymptotic probabilistic dynamics of the stochastically forced two-layer quasigeostrophic fluid system. We first transformed this system into a coupled system of random partial differential equations and then show that the asymptotic probabilistic dynamics of this system depends only on the top fluid layer, provided that the Wiener process and the fluid parameters satisfy a certain condition, i.e., the inequality (38); see Theorem 5.3. In particular, this latter condition is satisfied when the trace of the covariance operator of the Wiener process is controled by a certain upper bound (see Corollary 5.4) and the Ekman constant $r$ is sufficiently large (see the inequality (41)). Note that the generalized time derivative of the Wiener process models the fluctuating part of the wind stress forcing on the top fluid layer, and the Ekman constant $r$ measures the rate for vorticity decay due to the friction in the bottom Ekman layer.

Acknowledgement. A part of this work was done at the Oberwolfach Mathematical Research Institute, Germany, while J. Duan and B. Schmalfuß were Research in Pairs Fellows, supported by Volkswagen Stiftung. J. Duan would like to thank Tamay Ozgokmen, University of Miami, for helpful discussions. This work was partly supported by the NSF Grant DMS-9973204.

\section{REFERENCES}

[1] L. Arnold, Random Dynamical Systems. Springer, Berlin, 1998.

[2] L. Arnold, Hasselmann's programm visited: The analysis of stochasticity in deterministic climate models. Report 450, Universität Bremen, Institut für Dynamische Systeme, 2000.

[3] E. S. Benilov, On the stability of large-amplitude geostrophic flows in a two-layer fluid: the case of "strong" beta-effect. J. Fluid Mech. 284 (1995), 137-158.

[4] P. Berloff and S. P. Meacham, On the stability of equivalent-barotropic and baroclinic models of the wind-driven circulation, preprint, 1999.

[5] P. Berloff and J. C. McWilliams, Large-scale, low-frequency variability in wind-driven ocean gyres, J. Phys. Oceanogr. 29 (1999), 1925-1949.

[6] C. Bernier, Existence of attractor for the quasi-geostrophic approximation of the Navier-Stokes equations and estimate of its dimension. Advances in Mathematical Sciences and Applications, 4 (2), pp 465-489 (1994).

[7] Ch. Bernier-Kazantsev and I.D. Chueshov, The finiteness of determining degrees of freedom for the quasi-geostrophic multi-layer ocean model, Nonlinear Analysis 42 (2000), 1499-1512.

[8] L. Berselli and F. Flandoli. Remarks on determining projections for stochastic dissipative equations. Discrete and Continuous Dynamical Systems, 5(8):197-214, 1999.

[9] J. Brannan, J. Duan and T. Wanner, Dissipative Quasigeostrophic Dynamics under Random Forcing, J. Math. Anal. Appl. 228 (1998), 221-233.

[10] I. Chueshov, Theory of functionals that uniquely determine asymptotic dynamics of infinite-dimensional dissipative systems. Uspekhi Mat. Nauk, 53(4):77-124, 1998. (in Russian). English translation in Russian Mathematical Surveys 53:731-776, 1998. 
[11] I. Chueshov, Introduction to the Theory of Infinite-Dimensional Dissipative Systems. Acta, Kharkov, 1999. (in Russian).

[12] I. Chueshov, On determining functionals for stochastic Navier - Stokes equations. Stochastics and Stochastics Reports, 68:45-64, 1999.

[13] I. Chueshov, J. Duan and B. Schmalfuß. Determining functionals for random partial differential equations, To appear in Nonlinear Differential Equations and Applications, 2001.

[14] P. Constantin and C. Foias, Navier-Stokes Equations, Univ. of Chicago Press, Chicago, 1988.

[15] T. DelSole and B. F. Farrell, A stochastically excited linear system as a model for quasigeostrophic turbulence: Analytic results for one- and two-layer fluids, J. Atmos. Sci. 52 (1995) 2531-2547.

[16] V. Dymnikov and E. Kazantsev, On the genetic "memory" of chaotic attractor of the barotropic ocean model. In Proceedings of the third bilateral conference "Predictability of atmospheric and oceanic circulations " of the French-Russian A.M.Liapunov Institute in Computer Science and Applied Mathematics (INRIA - Moscow State University). Nancy, April, 1997.Edition MSU, 1997, pp. 25-36.

[17] F. Flandoli and J. A. Langa. On determining modes for dissipative random dynamical systems. Stochastics and Stochastics Reports, 66:1-25, 1999.

[18] C. Foias, O. Manley, R.Temam and Y.M. Treve, Asymptotic analysis of the NavierStokes equations, Physica D 9 (1983), 157-188.

[19] C. Foias and G. Prodi. Sur le comportement global des solutions nonstationnaires des équations de Navier-Stokes en dimension deux. Rend. Sem. Mat. Univ. Padova, 39:1-34, 1967.

[20] C. Foias, E.S. Titi, Determining nodes, finite difference schemes and inertial manifolds. Nonlinearity, 4, pp 135-153 (1991).

[21] A. Griffa and S. Castellari, Nonlinear general circulation of an ocean model driven by wind with a stochastic component, J. Marine Research 49 (1991), 53-73.

[22] K. Hasselmann. Stochastic climate models, Part I. Tellus, 28:473-485, 1976.

[23] G. Holloway, Ocean circulation: Flow in probability under statistical dynamical forcing, in Stochastic Models in Geosystems, S. Molchanov and W. Woyczynski (eds.), Springer, 1996.

[24] R. X. Huang and H. Stommel, Cross sections of a two-layer inertial Gulf Stream, J. Phys. Oceanography 20 (1990), 907-901.

[25] D.A. Jones and E.S. Titi, Determining finite volume elements for the 2D NavierStokes equations, Physica D 60 (1992), 165-174.

[26] O. Ladyzhenskaya. A dynamical system generated by the Navier-Stokes equations. Journal of Soviet Mathematics, 3:458-479, 1975.

[27] P. Müller, Stochastic forcing of quasi-geostrophic eddies, in Stochastic Modelling in Physical Oceanography, R. J. Adler, P. Müller and B. Rozovskii (eds.), Birkhäuser, 1996.

[28] T. Ozgokmen, Emergence of inertial gyres in a two-layer quasigeostrophic ocean model, J. Phys. Oceanography 28 (1998), 461-484.

[29] J. Pedlosky. Geophysical Fluid Dynamics. Springer Verlag, New-York, Berlin, 1987.

[30] J. Pedlosky, Ocean Circulation Theory. Springer-Verlag, Berlin, 1996.

[31] R. Salmon. Lectures on Geophysical Fluid Dynamics. Oxford Univ. Press, Oxford, 1998.

[32] R. Salmon. Generalized two-layer models of ocean circulation, J. Marine Research 52 (1994), 865-908.

[33] R. M. Samelson, Stochastically forced current fluctuations in vertical shear and over topography, J. Geophys. Res. 94 (1989) 8207-8215.

[34] W. M. Washington and C. L. Parkinson, An Introduction to Three-Dimensional Climate Modeling, Oxford Univ. Press, 1986. 
(Igor Chueshov) Institute für Dynamische Systeme, FB3, Universität Bremen, D-28334 Bremen, Germany. On leave from Department of Mechanics andMathEmatics, Kharkov University, 310077 Kharkov, Ukraine

E-mail address, Igor D. Chueshov: chueshov@math.uni-bremen.de

(Jinqiao Duan) Department of Applied Mathematics, Illinois Institute of TechNOLOGY, ChicAGo, IL 60616, USA.

E-mail address, Jinqiao Duan: duan@iit.edu

(Björn Schmalfuß) Department of Applied Sciences, University of Technology and Applied Sciences, Geusaer Strasse, D-06217 Merseburg, Germany,

E-mail address, Björn Schmalfuß: schmalfuss@in.fh-merseburg.de 